\title{
ON THE POSITIVE SQUARE ROOT OF THE FOURTH DERIVATIVE OPERATOR*
}

BY

\author{
D. L. RUSSELL ${ }^{1}$
}

University of Wisconsin, Madison, Wisconsin

Abstract. It is only for a special subset of the natural boundary conditions for the operator

$$
A w=\frac{d^{4} w}{d x^{4}}
$$

that its positive square root is the negative second derivative operator. In this paper we develop a procedure for parametric description of all natural boundary conditions, we show which ones admit $A^{1 / 2}$ in the form just noted, and we show that in the other cases

$$
-D^{2} w \equiv-\frac{d^{2} w}{d x^{2}}=[I+P] A^{1 / 2} w
$$

where $P$ is a bounded, but in general not compact, operator on the Hilbert space $L^{2}[0, \pi]$. Possible applications to the theory of the partial differential equation

$$
\rho \frac{\partial^{2} w}{\partial t^{2}}-2 \gamma \frac{\partial^{3} w}{\partial t \partial x^{2}}+E I \frac{\partial^{4} w}{\partial x^{4}}=0
$$

are indicated.

1. Introduction and statement of results. In [2] G. Chen and the author introduced the "square root damping" model

$$
\frac{d^{2} w}{d t^{2}}+2 \gamma A^{1 / 2} \frac{d w}{d t}+A w=0
$$

where $A^{1 / 2}$ is the nonnegative square root of the nonnegative, self-adjoint "elasticity" operator $A$, and $\gamma$ is a positive number, in order to account for observed relationships between damping rate and frequency in an elastic system with energy

$$
\mathscr{E}=\frac{1}{2}\left\{\left\|\frac{d w}{d t}\right\|^{2}+\left\|A^{1 / 2} w\right\|^{2}\right\},
$$

the norm being taken in an appropriate Hilbert space $W$ containing the system displacements $w$. The main example, and principal motivation for that paper, was the ${ }^{*}$ Received August 24, 1987.

${ }^{1}$ Supported in part by the U.S. Air Force of Scientific Research under Grant AFOSR 85-0263. Reproduction is permitted for U. S. Government purposes. 
case $W=L^{2}[0, \pi]$ and the "beam" operator defined (after normalization of physical constants) by

$$
(A w)(x)=\frac{d^{4} w}{d x^{4}}(x), \quad x \in(0, \pi),
$$

with $\mathscr{D}(A)$, the domain of $A$, a subspace of $H^{4}[0, \pi]$ characterized by boundary conditions for which $A$ is nonnegative and self-adjoint.

One of the disadvantages accompanying use of the model (1.01) lies in the physical interpretation of the damping operator $-2 \gamma A^{1 / 2}$. In Sec. 3 we classify all natural conditions under three distinct cases. In a particular subset of these cases, which might be called the "trigonometric cases", the nonnegative square root of $A$ is given by

$$
\left(A^{1 / 2} w\right)(x)=\left(-D^{2} w\right)(x)=-\frac{d^{2} w}{d x^{2}}(x)
$$

and, for a solution $w(x, t)$ of (1.01), interpreted in those cases as a partial differential equation, we have

$$
-2 \gamma A^{1 / 2} \frac{\partial w}{\partial t}=2 \gamma \frac{\partial^{3} w}{\partial t \partial x^{2}},
$$

so that the damping force is proportional to the bending rate, a very natural interpretation. For other boundary conditions, however, $A^{1 / 2}$ is not a differential operator and its interpretation is rather obscure. The purpose of this paper is to elucidate some of the properties of $A^{1 / 2}$ in those cases where it does not agree with the negative second derivative with a view to better understanding of the square root damping mechanism. In the process we encounter some rather interesting mathematics, some of historical interest, going back to Hardy, Littlewood, and Pòlya [4] and relating to the theory of Hankel operators on $\ell^{2}$ (see [9], e.g.).

Natural boundary conditions for (1.03) of spatially separated type are obtained by requiring $A$ to be self-adjoint on $L^{2}[0, \pi]$, here taken as a real Hilbert space. The symmetry condition is obtained by noting that, with $($,$) the inner product in$ $L^{2}[0, \pi]$,

$$
\begin{aligned}
(A w, v) & =\int_{0}^{\pi} w^{i \prime \prime}(x) v(x) d x \\
& =\left.\left[w^{\prime \prime \prime}(x) v(x)-w^{\prime \prime}(x) v^{\prime}(x)\right]\right|_{0} ^{\pi}+\int_{0}^{\pi} w^{\prime \prime}(x) v^{\prime \prime}(x) d x
\end{aligned}
$$

while

$$
\begin{aligned}
(w, A v) & =\int_{0}^{\pi} w(x) v^{i \prime \prime}(x) d x \\
& =\left.\left[w(x) v^{\prime \prime \prime}(x)-w^{\prime}(x) v^{\prime \prime}(x)\right]\right|_{0} ^{\pi}+\int_{0}^{\pi} w^{\prime \prime}(x) v^{\prime \prime}(x) d x
\end{aligned}
$$


It follows that $(A w, v)-(w, A v)=0$ for $w, v \in \mathscr{D}(A)$ just in case the boundary form (with $\hat{\mathbf{w}}$ denoting the vector with components $w, w^{\prime}$ etc.),

$$
\begin{aligned}
B(w, v) & =w^{\prime \prime \prime} v-w^{\prime \prime} v^{\prime}+w^{\prime} v^{\prime \prime}-w v^{\prime \prime \prime} \\
& =\left(w, w^{\prime}, w^{\prime \prime}, w^{\prime \prime \prime}\right)\left(\begin{array}{cccc}
0 & 0 & 0 & -1 \\
0 & 0 & 1 & 0 \\
0 & -1 & 0 & 0 \\
1 & 0 & 0 & 0
\end{array}\right)\left(\begin{array}{c}
v \\
v^{\prime} \\
v^{\prime \prime} \\
v^{\prime \prime \prime}
\end{array}\right) \equiv \hat{\mathbf{w}}^{*} \mathbf{B}_{0} \hat{\mathbf{v}},
\end{aligned}
$$

vanishes at 0 and $\pi$ when the boundary conditions apply there for both $w$ and $v$. Boundary conditions guaranteeing that $B(w, v)=0$ at $x=0$ and $x=\pi$ will be called "symmetric" boundary conditions in this paper. A subset of these boundary conditions consists of those which guarantee not only that $B(w, v)=0$ but also that, subject to these boundary conditions, the quadratic form

$$
w^{\prime} w^{\prime \prime}-w w^{\prime \prime \prime}=\frac{1}{2}\left(w, w^{\prime}, w^{\prime \prime}, w^{\prime \prime \prime}\right)\left(\begin{array}{cccc}
0 & 0 & 0 & -1 \\
0 & 0 & 1 & 0 \\
0 & 1 & 0 & 0 \\
-1 & 0 & 0 & 0
\end{array}\right)\left(\begin{array}{c}
w \\
w^{\prime} \\
w^{\prime \prime} \\
w^{\prime \prime \prime}
\end{array}\right) \equiv \hat{\mathbf{w}}^{*} \mathbf{C}_{0} \hat{\mathbf{w}}
$$

is nonpositive at $\pi$ and nonnegative at 0 so that (cf. (1.05)) the operator $A$ is nonnegative. We will call these "natural" boundary conditions here, perhaps departing from the standard usage in this respect. It can be seen that $A$ is self-adjoint and bounded below for all symmetric boundary conditions, natural or not. If the form (1.07) reduces to zero at both $x=0$ and $x=\pi$, the system energy becomes

$$
\frac{1}{2} \int_{0}^{\pi}\left(\left(\frac{\partial w}{\partial t}\right)^{2}+\left(\frac{\partial^{2} w}{\partial x^{2}}\right)^{2}\right) d x
$$

i.e., the energy is strictly distributed rather than a sum of this integral and boundary terms of the form (1.07). Natural boundary conditions for which the energy is strictly distributed will be referred to here as natural SDE boundary conditions. This set of boundary conditions includes clamped, free, hinged, and guided boundary conditions but excludes, e.g., "elastic" boundary conditions such as $u^{\prime \prime \prime}=a w$ ( $a>0$ if $x=\pi$, $a<0$ if $x=0$ ) for which (1.07) includes a term of the form $|a| u^{2}$ which is added to the indicated integral to form the complete system energy.

We will also have occasion in this paper to refer to boundary conditions satisfying the symmetry condition but such that the energy form (1.07) becomes nonpositive at $\pi$ and nonnegative at 0 . We will refer to boundary conditions of this type, for reasons soon to become apparent, as dual natural boundary conditions. Natural SDE boundary conditions are both natural and dual natural boundary conditions. While we will be interested only in natural boundary conditions which are independent of the eigenvalue parameter $\lambda$, the definitions remain valid if such a dependence does exist and, in fact, the dual natural boundary conditions which we have use for are in general of this type. More on this later.

Now we begin our systematic study of the difference between $A^{1 / 2}$ and the negative second derivative. Considering only natural boundary conditions henceforth 
in this section, it is well known that when $A$ is nonnegative its spectrum consists of eigenvalues

$$
0 \leq \lambda_{1}<\lambda_{2}<\cdots<\lambda_{k}<\lambda_{k+1}<\cdots,
$$

of single multiplicity when $\lambda_{k}$ is positive, with $\lim _{k \rightarrow \infty} \lambda_{k}=\infty$; in some cases the first, $\lambda_{1}$, is zero. When this is the case $\lambda_{1}$ may have multiplicity two but any corresponding eigenfunction $\varphi$ satisfies

$$
\begin{aligned}
0 & =\lambda_{1}(\varphi, \varphi)=(\varphi, A \varphi) \\
& =\left.\left[\varphi \varphi^{\prime \prime \prime}-\varphi^{\prime} \varphi^{\prime \prime}\right]\right|_{0} ^{\pi}+\int_{0}^{\pi}\left(\varphi^{\prime \prime}(x)\right)^{2} d x \geq \int_{0}^{\pi}\left(\varphi^{\prime \prime}(x)\right)^{2} d x
\end{aligned}
$$

so that $\varphi^{\prime \prime}(x)=0$ in $L^{2}[0, \pi]$ and $\varphi$ is, therefore, also an eigenfunction of the second derivative operator corresponding to the eigenvalue 0 . For this reason we will treat $\lambda_{k}>0$ in the sequel unless the contrary is explicitly stated.

The basic idea of our approach is to examine the differing actions of the second derivative operator on the real exponential and trigonometric parts of the eigenvectors, which we now proceed to describe.

Let $\varphi_{1}, \varphi_{2}, \varphi_{3}, \ldots$ be orthonormal eigenfunctions of $A$ corresponding to the eigenvalues $\lambda_{1}, \lambda_{2}, \lambda_{3}, \ldots$. These either form an orthonormal basis for $L^{2}[0, \pi]$ themselves or else may be modified to include a pair of orthonormalized eigenvectors corresponding to $\lambda_{1}$ when it is a zero eigenvalue of multiplicity two. For the positive $\lambda_{k}$ we let

$$
\lambda_{k}=\omega_{k}^{4}, \quad \omega_{k}>0,
$$

and, as we see from the form of the fourth-order operator $A=D^{4}$,

$$
\varphi_{k}(x)=\gamma_{k}^{+} e^{\omega_{k}(x-\pi)}+\gamma_{k}^{-} e^{-\left(\omega_{k} \cdot x\right.}+\gamma_{k}^{c} \cos \omega_{k} x+\gamma_{k}^{s} \sin \omega_{k} x
$$

for real coefficients as indicated. In $\S 3$ we establish the following lemma.

LemMA 1 . Let $\lambda$ be an eigenfunction of $A$ corresponding to an eigenvalue $\lambda=\omega^{4}>0$, $A$ being endowed with natural boundary conditions. Then

$$
\varphi(x)=\gamma^{+} e^{\omega(x-\pi)}+\gamma^{-} e^{-(\omega x}+\gamma^{c} \cos \omega x+\gamma^{s} \sin \omega x
$$

and there is a positive $\hat{\omega}$ and a positive $\hat{K}$ such that, for $\omega \geq \hat{\omega}$.

$$
\left(\gamma^{+}\right)^{2}+\left(\gamma^{-}\right)^{2} \leq \hat{K}\left[\left(\gamma^{c}\right)^{2}+\left(\gamma^{s}\right)^{2}\right] .
$$

As a consequence there is a positive number $K$ such that, for all values of $k$ in (1.10),

$$
\left(\gamma_{k}^{+}\right)^{2}+\left(\gamma_{k}^{-}\right)^{2} \leq K
$$

These elementary estimates are significant for the following reason. We let $A^{1 / 2}$ denote the nonnegative square root of $A, A$ endowed with natural boundary conditions, and we let $-D^{2}$ denote the negative second derivative operator $-d^{2} / d x^{2}$. Then an elementary calculation shows that, for $\lambda_{k}>0$,

$$
\begin{aligned}
\left(D \varphi_{k}\right)(x) & =-2 \omega_{k}^{2}\left[\gamma_{k}^{+} e^{\left(\omega_{k}(x-\pi)\right.}+\gamma_{k}^{-} e^{-()_{k} \cdot x}\right]+\omega_{k}^{2} \varphi_{k}(x) \\
& \equiv \omega_{k}^{2} \psi_{k},
\end{aligned}
$$


while, clearly,

$$
A^{1 / 2} \varphi_{k}=\omega_{k}^{2} \varphi_{k}, \quad k=1,2,3, \ldots
$$

We tentatively define an operator $P$ for $\varphi_{k}$ such that $\lambda_{k}>0$ by

$$
\left(P \varphi_{k}\right)(x)=-2\left(\gamma_{k}^{+} e^{\omega_{k}(x-\pi)}+\gamma_{k}^{-} e^{-\left(\omega_{k} \cdot x\right.}\right),
$$

and, where a zero eigenvalue $\lambda_{1}$ exists, $P \varphi_{1}=-\varphi_{1}$ for any eigenvector $\varphi_{1}$ associated with $\lambda_{1}$. If we let $\mathbb{P}=I+P$, we have (cf. (1.14))

$$
\mathbb{P} \varphi_{k}=\psi_{k}, \quad k=1,2,3, \ldots,
$$

provided $\varphi_{k}$ is such that $\lambda_{k}>0$, while $\mathbb{P} \varphi_{1}=0$ when $\varphi_{1}$ is a zero eigenvector.

From (1.13) and the fact that the $\varphi_{k}$ form an orthonormal basis for $L^{2}[0, \pi]$, the boundedness of $P$ is obtained as stated in the following theorem. The indicated asymptotic form of the $\omega_{k}$ is too familiar to require proof but a brief sketch of how such a proof might be carried out is given in $\S 3$.

THEOREM 2. Endowed with natural boundary conditions, the operator $A$ of (1.03) has eigenvalues $\lambda_{k}$ such that the $\omega_{k}$ defined in (1.09) have the form

$$
\omega_{k}=k+\nu+\varepsilon_{k}, \quad\left\{\varepsilon_{k}\right\} \in \ell^{2},
$$

and, as a consequence of this and the result of Lemma 1, the operator $P$ defined in (1.16) extends to a bounded but, in general, noncompact operator on $L^{2}[0, \pi]$.

The main result proved in this paper, the first part of which follows from Theorem 2 (but, in fact, is reproved independently in $\S 3$ ) is

THEOREM 3. The negative second derivative operator $-D^{2}$ of $(1.14)$, with domain $\mathscr{D}$ the same as that of $A^{1 / 2}$, the nonnegative square root of the fourth-order operator $A$ of (1.03) (assuming natural boundary conditions so that $A$ is nonnegative), has the form

$$
-D^{2} w=\mathbb{P} A^{1 / 2} w, \quad w \in \mathscr{D},
$$

where $\mathbb{P}$ is the operator defined in (1.17). The subspace $\mathscr{R} \subset L^{2}[0, \pi]$ which is the range of $-D^{2}$ is spanned by the $\psi_{k}$ corresponding to $\lambda_{k}>0$ and is closed in $L^{2}[0, \pi]$. The operator $\mathbb{P}$ has a bounded inverse on $\mathscr{R}$, which extends to a bounded operator $\mathbb{Q}$ on $L^{2}[0, \pi]$, so that likewise

$$
A^{1 / 2} w=\mathbb{Q}\left(-D^{2}\right) w, \quad w \in \mathscr{D} .
$$

The boundedness of $P$ as a consequence of $(1.18)$ is proved in $\S 2$. This is equivalent to the boundedness of $\mathbb{P}$ but the latter is also proved by a different method in $\S 3$, as we have remarked earlier.

One should note that $\mathbb{P}$ depends more or less exclusively on the boundary conditions imposed on $A$, not only because these influence the values of the $\omega_{k}$ but also because it becomes the identity whenever those boundary conditions are such as to ensure purely trigonometric eigenvectors for $A$ corresponding to eigenvalues $\lambda_{k}>0$.

Using [6], (pp. $487 \mathrm{ff}$.) we immediately see that the partial differential equation,

$$
\rho \frac{\partial^{2} w}{\partial t^{2}}-2 \gamma \frac{\partial^{3} w}{\partial t \partial x^{2}}+E I \frac{\partial^{4} w}{\partial x^{4}}=0,
$$


which we may rewrite in operator terminology as

$$
\rho \ddot{w}-2 \gamma D^{2} \dot{w}+E I A w=0,
$$

corresponds to a first-order system associated with a holomorphic semigroup in the Hilbert space with norm equivalent to the physical energy of the beam system, provided that the bounds on the coefficients $\gamma_{k}^{+}, \gamma_{k}^{-}$are sufficiently small, so that the norm of $P$ is also sufficiently small. If $P$ were compact, and we have noted that this is, in fact, not the case, we could draw this conclusion without reservation. We are studying this partial differential equation via other techniques and expect to report improved results shortly.

It is possible to make a fairly substantial improvement on the boundedness of $P$ stated in Theorem 2. Although the strengthened result is not necessary for the study of the operators $P$ described in (1.16), it seems worthwhile to state it here for its own sake. A rather similar result, which we do not detail here, is part of Theorem 1.6 of [9], but only for $T^{*} T$ a Hankel operator, that is, an infinite matrix operator on $\ell^{2}$ whose $i, j$-th entry is $a_{i+j}$ for some sequence $\left\{a_{k}\right\}$ with particular properties. The present result bears no such restriction.

THEOREM 4. Let $\left\{\omega_{k}\right\}$ be a sequence of positive numbers such that

$$
k / \omega_{k} \leq B, \quad k=1,2,3, \ldots,
$$

for some fixed positive constant $B$. Then the operator $T: /^{2} \rightarrow L^{2}[0, \pi]$ defined by

$$
T\left\{c_{k}\right\}=\sum_{k=1}^{\infty} e^{-\omega_{k} \cdot x}
$$

is bounded.

The proof of this result will also be given in $\S 2$. These theorems clearly relate to exponential sequences which, according to the classical Muntz-Szasz theory (see, e.g., [7]) are complete in $L^{2}[0, \pi]$. It follows that the range of $T$, or $P$, is dense in $L^{2}[0, \pi]$. We should also remark that it is possible to obtain these boundedness results with the use of the Carleson measure theorem ([3], [5]), but this seems rather in the nature of mathematical overkill in this situation.

2. Boundedness and noncompactness of $P$; Proof of Theorem 4. The asymptotic properties, as $k \rightarrow \infty$, of the numbers $\omega_{k}=\lambda_{k}^{1 / 4}$ described in (1.09) are too well known to require any formal treatment here. However, the methods of $\S 3$ allow one to formulate certain trigonometric/exponential equations whose roots are the $\omega_{k}$, from which it may be seen that, for $k=1,2,3, \ldots$,

$$
\omega_{k}=k+\nu+\varepsilon_{k}, \quad\left\{\varepsilon_{k}\right\} \in \digamma^{2},
$$

where the value of the constant $\nu$ depends on the specific boundary conditions applied, as does the particular sequence $\left\{\varepsilon_{k}\right\}$. Using (2.01) along with the form (1.16) of the operator $P$, we see that the properties of $P$, such as boundedness, compactness, etc., must be the same as those of the operator

$$
T:\left\{c_{k}\right\} \in \ell^{2} \rightarrow \sum_{k=1}^{\infty} c_{k} e^{-()_{k} x} \in L^{2}[0, \pi] .
$$


(That $A$ might have a zero eigenvalue with accompanying $\omega_{0}=0$ does not affect this conclusion.)

We will show, first of all, that $T$ is bounded. This can be established by use of an inequality appearing in [4], the usefulness of which reference was conveyed to the author by R. J. Duffin and is here gratefully acknowledged, and by another method which appears at the end of this section. As the first method, though less general, is best adapted to the later investigation of compactness, and since the treatment in [4] is rather cursory, we will take some pains to develop the result here.

Clearly

$$
T\left\{c_{k}\right\}=e^{-\nu x}\left\{\sum_{k=1}^{\infty} c_{k} e^{-k x}+\sum_{k=1}^{\infty} c_{k}\left(e^{-\left(k+\varepsilon_{k}\right) x}-e^{-k \cdot x}\right)\right\}
$$

and the fixed factor $e^{-\nu x}$ does not affect any properties of $T$ of interest to us here. We note that

$$
\begin{aligned}
\left\|e^{-\left(k+\varepsilon_{k}\right) x}-e^{-k x}\right\|_{L^{2}[0, \pi]}^{2} & =\int_{0}^{\pi} e^{-2 k x}\left|e^{-\varepsilon_{k} x}-1\right|^{2} d x \\
& \leq \tilde{\mu}^{2}\left|\varepsilon_{k}\right|^{2} \int_{0}^{\pi} e^{-2 k x} d x \\
& \leq \mu^{2} \frac{\left|\varepsilon_{k}\right|^{2}}{k}
\end{aligned}
$$

for some positive constants $\mu, \tilde{\mu}$. Defining the operator

$$
\delta T\left\{c_{k}\right\} \equiv \sum_{k=1}^{\infty} c_{k}\left(e^{-\left(k+\varepsilon_{k}\right) x}-e^{-k x}\right)
$$

the Minkowski and Schwarz inequalities yield

$$
\left\|\delta T\left\{c_{k}\right\}\right\|_{L^{2}[0, T]} \leq \mu \sqrt{\sum_{k=1}^{\infty}\left|\varepsilon_{k}\right|^{2}}\left\|\left\{\frac{c_{k}}{k^{1 / 2}}\right\}\right\|_{/ 2} \leq \mu\left\|\left\{\varepsilon_{k}\right\}\right\|_{/=}\left\|\left\{c_{k}\right\}\right\|_{/:} .
$$

Thus $T$ is bounded if and only if $T_{0}$, defined by

$$
T_{0}\left\{c_{k}\right\}=\sum_{k=1}^{\infty} c_{k} e^{-k x}, \quad\left\{c_{k}\right\} \in \ell^{2}
$$

defines a bounded operator from $\ell^{2}$ to $L^{2}[0, \pi]$.

It is clear that an even stronger result is obtained if we replace $L^{2}[0, \pi]$ by $L^{2}[0, \infty)$. Then, making the change of independent variable $z=e^{-x}$ and noting that, for $f \in L^{2}[0, \infty), \tilde{f}(z)=f(-\log z)$,

$$
\int_{0}^{\infty}|f(x)|^{2} d x=\int_{0}^{1} \frac{|\tilde{f}(z)|^{2} d z}{z}
$$

we see that we may consider the convergence of

$$
\sum_{k=1}^{\infty} c_{k} z^{k}
$$


in the function space whose squared norm is defined by the right-hand side of (2.05). Since it is clearly adequate to treat the case wherein the $c_{k}$ are all nonnegative, we assume the latter and study the weighted square sum

$$
\frac{1}{z}\left(\sum_{k=1}^{\infty} c_{k} z^{k}\right)^{2}, \quad\left\{c_{k}\right\} \in \nearrow^{2} .
$$

We may then suppose

$$
0<c_{k} \leq c
$$

for some $c>0$. We dominate the sum (2.06), clearly convergent for $|z|<1$, on the segment $(-1,0]$ by

and conclude that

$$
c \sum_{k=1}^{\infty}(-1)^{k} z^{k}=\frac{-c z}{1+z}
$$

$$
(1+z)^{2}\left(\sum_{k=1}^{\infty} c_{k} z^{k}\right)^{2} \equiv g(z)
$$

is uniformly bounded for $-1 \leq z \leq 1$. Integrating $g(z)$ over positively oriented contours $\Gamma_{r}, 0<r<1$, consisting of the real axis from $z=-r$ to $z=r$ and the half circle

Cauchy's theorem gives

$$
C_{r}^{+}=\{z|| z \mid=r, \operatorname{Im}(z)>0\},
$$

$$
\int_{0}^{r} g(z) d z=-\int_{-r}^{0} g(z) d z+\int_{C ;} g(z) d z .
$$

Since the first term on the right-hand side is negative, the second is positive and

$$
\int_{0}^{r} g(z) d z \leq \int_{C_{i}^{\prime}} g(z) d z \leq \int_{C_{i}}|z||g(z)| d \theta,
$$

where $C_{r}$ is the positively oriented circle of radius $r$. Clearly

Then we compute that

$$
\int_{C_{r}}\left|\sum_{k=1}^{\infty} c_{k} z^{k}\right|^{2} d \theta=2 \pi \sum_{k=1}^{\infty}\left|c_{k}\right|^{2} r^{2 k} .
$$

$$
\begin{aligned}
\int_{0}^{1} \frac{1}{z}\left(\sum_{k=1}^{\infty} c_{k} z^{k}\right)^{2} d z & \leq \int_{0}^{1} \frac{(1+z)^{2}}{z}\left(\sum_{k=1}^{\infty} c_{k} z^{k}\right)^{2} d z \\
& =\int_{0}^{1} \frac{g(z)}{z} d z \\
& \leq \lim _{r \rightarrow \infty} \int_{c_{i}}|g(z)| d \theta \\
& \leq 4 \lim _{r \rightarrow \infty} \int_{c_{i}}\left|\sum_{k=1}^{\infty} c_{k} z^{k}\right|^{2} d \theta \\
& =8 \pi \sum_{k=1}^{\infty}\left|c_{k}\right|^{2},
\end{aligned}
$$


so that

$$
\left\|T_{0}\right\| \leq 2 \sqrt{2 \pi}
$$

and thus, noting our previous arguments, $T$, and hence $P$, are bounded operators as well.

Since the operator on $\ell^{2}$ which carries the sequence $\left\{c_{k}\right\}$ to $\left\{c_{k} / \sqrt{k}\right\}$ is clearly compact, the operator $\delta T$ in (2.03) is compact and thus $T$ is compact if and only if $T_{0}$ in (2.04) is compact. It is then a simple matter to see that $P$ is compact if and only if $T_{0}$ is compact.

Now, in fact, none of these operators are compact. It is just a matter of showing that $T_{0}$ is not. Since the map $T_{\pi}:\left\{c_{k}\right\} \in \ell^{2} \rightarrow \sum_{k=1}^{\infty} c_{k} e^{-k \cdot r} \in L^{2}[\pi, \infty)$ is easily seen to be compact, we may again treat $T_{0}$ as a map from $/^{2}$ to $L^{2}[0, \infty)$. The noncompactness may then be established by various means. One may study, e.g., the Hankel operator $T_{0}^{*} T_{0}: \ell^{2} \rightarrow \ell^{2}$ and cite the result in [9] (Theorem 1.6, pp. 7, 8). However, to make this paper self-contained, we offer the following very simple argument communicated to the author by Professor A. Nagel.

The Hilbert space $\ell^{2}$, with sequences indexed $k=1,2,3, \ldots$, is isometric with the closed subspace $H_{1}^{2}$ of $H^{2}(D)$, the Hardy space in the interior of the unit disc $D$ with norm

$$
\|h\|^{2}=\frac{1}{2 \pi} \int_{i D}|h(z)|^{2} d|z|,
$$

spanned by the functions $z, z^{2}, z^{3}, \ldots$ Again using the change of independent variable $z=e^{-x}$, we may identify $T_{0}$ with the map from $h \in H_{1}^{2}$ to the restriction of $h$ to the real segment $0 \leq z<1$ in the space with squared norm (2.05). Consider the functions

$$
\hat{h}_{a}(z)=\frac{z}{a-z}, \quad a>1
$$

in $H_{1}^{2}$. Since

$$
\hat{h}_{a}(z)=\sum_{k=1}^{\infty} \frac{z^{k}}{a^{k}}
$$

its norm in $H^{2}(D)$ is

$$
\sqrt{\sum_{k=1}^{\infty} \frac{1}{a^{2 k}}}=\frac{1}{\sqrt{a^{2}-1}}
$$

and it follows that

$$
h_{a}(z)=\sqrt{a^{2}-1} \frac{z}{a-z}, \quad a>1,
$$

has unit norm in $H^{2}(D)$ for each such $a$. Letting $\left\{a_{k}\right\}$ be a sequence converging to 1 from above, the compactness of $T_{0}$ would imply the convergence, relative to the norm (2.05), of a subsequence $\left\{h_{a_{k}}\right\}$, which we may still call $\left\{h_{a_{k}}\right\}$. Since

$$
\lim _{a_{h} \downarrow 1} \frac{h_{a}(z)^{2}}{z}=0
$$


uniformly on every interval $[0,1-\delta], \delta>0$, the only possible limit is the zero function. But

$$
\begin{aligned}
\int_{0}^{1} \frac{\left(a^{2}-1\right)}{z} \hat{h}_{a}(z)^{2} d z & =\left(a^{2}-1\right)\left[\int_{0}^{1} \frac{z d z}{(a-z)^{2}}+a \int_{0}^{1} \frac{d z}{(z-a)^{2}}\right] \\
& =\left(a^{2}-1\right)\left[\log |a-1|-\log a+a\left(\frac{1}{a-1}-\frac{1}{a}\right)\right] \\
& =\left(a^{2}-1\right)\left[\log |a-1|-\log a+\frac{1}{a-1}\right],
\end{aligned}
$$

from which it is clear that

$$
\lim _{a_{k} \downarrow 1} \int_{0}^{1} \frac{h_{a}(z)^{2}}{z} d z=\lim _{a_{k} \downarrow 1}\left(a_{k}+1\right)=2
$$

and hence $\left\{h_{a_{k}}\right\}$ cannot converge, in the indicated norm, to 0 . It follows that $T_{0}$ is not compact and, as argued earlier, $P$ is likewise not compact if all that can be assumed is the boundedness of $\gamma_{k}^{+}$and $\gamma_{k}^{-}$as established in $\S 3$.

Proof of Theorem 4. Assume that $\left\{c_{k}\right\}$ is a finite sequence so that the right-hand side of $(1.20)$ lies in $L^{2}[0, \pi]$ without question. An arbitrary element $g \in L^{2}[0, \pi]$ may be represented by the series

$$
g(x)=\sum_{\ell=1}^{\infty} d_{\ell} \sin \ell x, \quad\left\{d_{\ell}\right\} \in \ell^{2},
$$

convergent in $L^{2}[0, \pi]$. A familiar computation shows that

$$
\int_{0}^{\pi} \sin \ell x e^{-\omega x} d x=\frac{\ell-\ell e^{-\omega \pi} \cos \ell \pi}{\omega^{2}+\ell^{2}}
$$

from which we conclude that, with $h=T\left\{c_{k}\right\}$,

$$
(h, g)_{L^{2}[0, \pi]}=\sum_{k, \ell} \frac{\ell c_{k} \overline{g_{\ell}}}{\omega^{2}+\ell^{2}}\left(1-e^{-\omega_{k} \pi} \cos \ell \pi\right) .
$$

Using (1.19) and assuming, without loss of generality, that $B \geq 1$,

$$
|(h, g)| \leq 2 B^{2} \sum_{k, \zeta} \frac{\ell\left|c_{k}\right||g /|}{\omega_{k}^{2}+\ell^{2}} .
$$

On the square $k \leq x \leq k+1, \ell \leq y \leq \ell+1$ we define

$$
\begin{gathered}
\tilde{c}(\xi)=c_{k}, \quad k \geq 1, \quad \tilde{c}(\xi)=0, \quad k=0, \\
\tilde{g}(\eta)=g_{\ell}, \quad \ell \geq 1, \quad \tilde{g}(\eta)=0, \quad \ell=0,
\end{gathered}
$$

and we observe that, on that square, for $k^{2}+\ell^{2} \geq 1$,

$$
k^{2}+\ell^{2} \geq \frac{1}{4}\left(\xi^{2}+\eta^{2}\right), \quad \ell \leq \eta,
$$

so that

$$
|(h, g)| \leq 8 B^{2} \int_{0}^{\infty} \int_{0}^{\infty} \frac{\eta \tilde{c}(\xi) \tilde{g}(\eta) d \xi d \eta}{\xi^{2}+\eta^{2}}
$$


Passing to polar coordinates $\xi=\rho \cos \theta, \eta=\rho \sin \theta$, we must estimate the integral

$$
\int_{0}^{\pi / 2} \int_{0}^{\infty} \sin \theta \tilde{c}(\rho \cos \theta) \tilde{g}(\rho \sin \theta) d \rho d \theta .
$$

Return to $\eta=\eta(\theta)=\rho \sin \theta$ in the inner integral and we have

$$
\int_{0}^{\pi / 2} \int_{0}^{\infty} \tilde{c}(\eta(\theta) \cot \theta) \tilde{g}(\eta(\theta)) d \eta(\theta) d \theta .
$$

Application of the Schwarz inequality yields

$$
\begin{aligned}
& \mid \int_{0}^{\infty} \tilde{c}(\eta(\theta) \cot \theta) \tilde{g}(\eta(\theta)) d \eta(\theta) \mid \\
& \quad \leq \sqrt{\int_{0}^{\infty} \tilde{g}(\eta)^{2} d \eta} \sqrt{\int_{0}^{\infty} \tilde{c}(\eta \cot \theta)^{2} d \eta} \\
& \quad=\|\tilde{g}\|_{L^{2}[0, \infty)}(\tan \theta)^{1 / 2} \sqrt{\int_{0}^{\infty} \tilde{c}(\eta \cot \theta)^{2} d(\eta \cot \theta)} \\
& \quad=(\tan \theta)^{1 / 2}\|g\|_{L^{2}[0, \infty)}\|\tilde{c}\|_{L^{2}[0, \infty)} .
\end{aligned}
$$

Since $(\tan \theta)^{1 / 2}$ is integrable on $[0, \pi / 2]$ we have, for some $\beta>0$,

$$
|(h, g)| \leq \beta\|\tilde{g}\|_{L^{2}[0, \infty)}\|\tilde{c}\|_{L^{2}[0, \infty)}=\frac{2 \beta}{\pi}\|g\|_{L^{2}[0, \pi]}\left\|\left\{c_{k}\right\}\right\|_{r} .
$$

Then, since we may take $g=h=T\left\{c_{k}\right\}$, we have

$$
\left\|T\left\{c_{k}\right\}\right\|_{L^{2}[0, \pi]} \leq \frac{2 \beta}{\pi}\left\|\left\{c_{k}\right\}\right\|_{/ 2 .}
$$

Approximating arbitrary $\left\{c_{k}\right\} \in \ell^{2}$ by finite sequences in the usual way we see that $\|T\| \leq 2 \beta / \pi$ and the theorem is proved.

3. Proof of Theorem 3. We begin with the system of differential equations satisfied by the eigenfunctions, written in the form

$$
\frac{d \hat{\mathbf{w}}}{d x}=\frac{d}{d x}\left(\begin{array}{c}
w \\
w^{\prime} \\
w^{\prime \prime} \\
w^{\prime \prime \prime}
\end{array}\right)=\left(\begin{array}{cccc}
0 & 1 & 0 & 0 \\
0 & 0 & 1 & 0 \\
0 & 0 & 0 & 1 \\
\omega^{4} & 0 & 0 & 0
\end{array}\right)\left(\begin{array}{c}
w \\
w^{\prime} \\
w^{\prime \prime} \\
w^{\prime \prime \prime}
\end{array}\right)=\mathbf{S}_{0}(\omega) \hat{\mathbf{w}}
$$

The boundary form is, from (1.06),

$$
\left(w, w^{\prime}, w^{\prime \prime}, w^{\prime \prime \prime}\right)\left(\begin{array}{cccc}
0 & 0 & 0 & -1 \\
0 & 0 & 1 & 0 \\
0 & -1 & 0 & 0 \\
1 & 0 & 0 & 0
\end{array}\right)\left(\begin{array}{c}
v \\
v^{\prime} \\
v^{\prime \prime} \\
v^{\prime \prime \prime}
\end{array}\right) \equiv \hat{\mathbf{w}}^{*} \mathbf{B}_{0} \hat{\mathbf{v}},
$$

while the energy form is $\hat{\mathbf{w}}^{*} \mathbf{C}_{0} \hat{\mathbf{v}}$, with $\mathbf{C}_{0}$ as given by (1.07). We introduce the transformation

$$
\hat{\mathbf{w}}=\mathbf{P}(\omega) \tilde{\mathbf{w}}, \quad \hat{\mathbf{v}}=\mathbf{P}(\omega) \tilde{\mathbf{v}}
$$


where

$$
\mathbf{P}(\omega)=\frac{1}{2}\left(\begin{array}{cccc}
1 & 1 & -1 & 0 \\
-\omega & \omega & 0 & -\omega \\
\omega^{2} & \omega^{2} & \omega^{2} & 0 \\
-\omega^{3} & \omega^{3} & 0 & \omega^{3}
\end{array}\right)
$$

The result is a transformed system

$$
\frac{d \tilde{\mathbf{w}}}{d x}=\mathbf{S}_{1}(\omega) \tilde{\mathbf{w}}
$$

wherein

$$
\mathbf{S}_{1}(\omega)=\left(\begin{array}{cccc}
-\omega & 0 & 0 & 0 \\
0 & \omega & 0 & 0 \\
0 & 0 & 0 & \omega \\
0 & 0 & -\omega & 0
\end{array}\right)
$$

a transformed boundary expression

$$
\tilde{\mathbf{w}}^{*} \mathbf{P}(\omega)^{*} \mathbf{B}_{0} \mathbf{P}(\omega) \tilde{\mathbf{v}} \equiv \tilde{\mathbf{w}}^{*} \mathbf{B}_{1}(\omega) \tilde{\mathbf{v}}
$$

with

$$
\mathbf{B}_{1}(\omega)=\omega^{3}\left(\begin{array}{cccc}
0 & -1 & 0 & 0 \\
1 & 0 & 0 & 0 \\
0 & 0 & 0 & 1 / 2 \\
0 & 0 & -1 / 2 & 0
\end{array}\right) \equiv \omega^{3}\left(\begin{array}{cc}
\mathbf{J} & 0 \\
\mathbf{0} & -\frac{1}{2} \mathbf{J}
\end{array}\right)
$$

and a transformed energy expression (cf. (1.07))

$$
\tilde{\mathbf{w}}^{*} \mathbf{P}(\omega)^{*} \mathbf{C}_{0} \mathbf{P}(\omega) \tilde{\mathbf{v}} \equiv \tilde{\mathbf{w}}^{*} \mathbf{C}_{1}(\omega) \tilde{\mathbf{v}}
$$

with

$$
\mathbf{C}_{1}(\omega)=\frac{1}{2} \omega^{3}\left(\begin{array}{cccc}
0 & 0 & 1 & 1 \\
0 & 0 & -1 & 1 \\
1 & -1 & 0 & 0 \\
1 & 1 & 0 & 0
\end{array}\right) \equiv \frac{1}{2} \omega^{3}\left(\begin{array}{cc}
\mathbf{0} & \mathbf{K} \\
\mathbf{K}^{*} & \mathbf{0}
\end{array}\right)
$$

In the $\tilde{\mathbf{w}}$ variables it is immediate that the general solution of (3.05) takes the form

$$
\tilde{\mathbf{w}}(x, \omega)=\left(\begin{array}{cccc}
e^{-\omega x} & 0 & 0 & 0 \\
0 & e^{\omega x} & 0 & 0 \\
0 & 0 & \cos \omega x & \sin \omega x \\
0 & 0 & -\sin \omega x & \cos \omega x
\end{array}\right) \tilde{\mathbf{w}}(0, \omega) \equiv \mathcal{J}^{\prime}(x,(1)) \tilde{\mathbf{w}}(0,(1))
$$

or, equally well,

$$
\tilde{\mathbf{w}}(x, \omega)=\mathscr{P}(x-\pi, \omega) \tilde{\mathbf{w}}(\pi, \omega) .
$$

The first and second components of the vector $\tilde{\mathbf{w}}$ (or $\tilde{\mathbf{v}}$ ) constitute the exponential part of $\tilde{\mathbf{w}}$, which we denote by $\tilde{\mathbf{w}}_{\boldsymbol{e}}$, while the third and fourth components constitute the trigonometric part $\tilde{\mathbf{w}}_{l}$.

Another proof of the boundedness of the operator $\mathbb{P}$. We have noted in Section 1 , from the form taken by the operator $P$, that its action is just to change the signs of the coefficients $\gamma^{+}$and $\gamma^{-}$in the representation

$$
\varphi(x)=\gamma^{+} e^{(1)(x-\pi)}+\gamma^{-} e^{-(1) x}+\gamma^{\prime} \cos \omega x+\gamma^{s} \sin (1) x
$$


of an eigenfunction of $A$ corresponding to the eigenvalue $\lambda=\omega^{4}$. From the form of the transformation (3.04) we see that the first component of $\hat{\mathbf{w}}(x)(\equiv \hat{\varphi}(x))$, in this case the value of $\varphi(x)$ itself, is given by

$$
\varphi(x)=\tilde{\mathbf{w}}^{1}(0, \omega) e^{-\omega x}+\tilde{\mathbf{w}}^{2}(0, \omega) e^{\omega x}-\tilde{\mathbf{w}}^{3}(0, \omega) \cos \omega x-\tilde{\mathbf{w}}^{4}(0, \omega) \sin \omega x
$$

so that

$$
\gamma^{-}=\tilde{\mathbf{w}}^{1}(0, \omega), \quad \gamma^{+}=\tilde{\mathbf{w}}^{2}(0, \omega) e^{(j) \pi}=\tilde{\mathbf{w}}^{2}(\pi, \omega) .
$$

It follows that changing the signs of $\gamma^{+}$and $\gamma^{-}$simply corresponds to changing the sign of the exponential part of $\tilde{\mathbf{w}}$, i.e., $\tilde{\mathbf{w}}_{c}$. To appreciate the consequences of this observation it is necessary to consider the boundary conditions in more detail.

To do this, we note, following [1], [8], [10], that instead of giving the boundary conditions in linear functional form, as one usually does, we may, equivalently, indicate the subspaces to which the boundary values and derivatives of $w($ or $\varphi)$ belong. In terms of the $\tilde{\mathbf{w}}$ variables we may represent the boundary subspaces in the form

$$
\tilde{\mathbf{w}}(0, \omega)=\mathbf{D z}, \quad \tilde{\mathbf{w}}(\pi, \omega)=\Delta \boldsymbol{\zeta},
$$

where $\mathbf{D}$ and $\Delta$ are $4 \times 2$, rank 2 basis matrices (i.e., their columns span the corresponding spaces) at $x=0$ and $x=\pi$, respectively, and $\mathbf{z}, \zeta$ are arbitrary vectors in $\mathbf{R}^{2}$. We may represent the upper and lower halves of $\mathbf{D}, \Delta$ conformally with $\tilde{\mathbf{w}}_{c}$, and $\tilde{\mathbf{w}}_{t} ;$ i.e.,

$$
\mathbf{D}=\left(\begin{array}{c}
\mathbf{D}_{e} \\
\mathbf{D}_{l}
\end{array}\right), \quad \Delta=\left(\begin{array}{c}
\boldsymbol{\Delta}_{\mathcal{c}} \\
\boldsymbol{\Delta}_{l}
\end{array}\right)
$$

The symmetry condition (3.02) on the operator $A$, i.e.,

$$
0=(A w, v)-(w, A v)=\left.\hat{\mathbf{w}}^{*} \mathbf{B}_{0} \hat{\mathbf{v}}\right|_{0} ^{\pi}=\left.\tilde{\mathbf{w}}^{*} \mathbf{B}_{1} \tilde{\mathbf{v}}\right|_{0} ^{\pi} .
$$

becomes, since we assume separated boundary conditions,

$$
\boldsymbol{\zeta}^{*} \Delta^{*} \mathbf{B}_{1} \Delta \boldsymbol{\eta}=0, \quad \mathbf{z}^{*} \mathbf{D}^{*} \mathbf{B}_{1} \mathbf{D} \mathbf{y}=0,
$$

where $\boldsymbol{\eta}$ and $\mathbf{y}$ are related to $\tilde{\mathbf{v}}(\pi, \omega)$ and $\tilde{\mathbf{v}}(0, \omega)$ in the same way as $\zeta$ and $\mathbf{z}$ are related to the corresponding $\tilde{\mathbf{w}}$ values. Now taking (3.14) and the form (3.08) of $\mathbf{B}_{1}$ into account, these conditions take the form

$$
\mathbf{D}_{e}^{*} \mathbf{J D}_{c}-\frac{1}{2} \mathbf{D}_{t}^{*} \mathbf{J} \mathbf{D}_{l}=\mathbf{0}, \quad \Delta_{l}^{*} \mathbf{J} \Delta_{l}-\frac{1}{2} \Delta_{l}^{*} \mathbf{J} \Delta_{l}=\mathbf{0} .
$$

Similarly, the positivity, at $x=0$, of the boundary energy form (cf. (3.10)) for $A$ becomes

$$
\mathbf{D}_{\mathcal{c}}^{*} \mathbf{K} \mathbf{D}_{t}-\mathbf{D}_{l}^{*} \mathbf{K D}_{\boldsymbol{c}^{\prime}} \leq 0, \quad \boldsymbol{\Delta}_{c^{\prime}}^{*} \mathbf{K} \boldsymbol{\Delta}_{t}-\boldsymbol{\Delta}_{l}^{*} \mathbf{K} \boldsymbol{\Delta}_{c^{\prime}} \geq 0 .
$$

In the $\hat{\mathbf{w}}$ variables we similarly have

$$
\hat{\mathbf{w}}(0, \omega)=\mathbf{E z}, \quad \hat{\mathbf{w}}(\pi, \omega)=\boldsymbol{\varepsilon} \boldsymbol{\zeta}, \quad \mathbf{E}=\mathbf{P}(\omega) \mathbf{D}, \quad \boldsymbol{\varepsilon}=\mathbf{P}((1)) \boldsymbol{\Delta} .
$$

and we can represent $\mathbf{E}, \boldsymbol{\varepsilon}$ in partitioned form as

$$
\mathbf{E}=\left(\begin{array}{l}
\mathbf{E}_{1} \\
\mathbf{E}_{2}
\end{array}\right), \quad \varepsilon=\left(\begin{array}{l}
\boldsymbol{\varepsilon}_{1} \\
\boldsymbol{\varepsilon}_{2}
\end{array}\right)
$$


where the $\mathbf{E}_{i}, \boldsymbol{\varepsilon}_{i}$, are $2 \times 2$ matrices. Let us represent the change of sign of the exponential part in the $\tilde{\mathbf{w}}$ variables by

$$
\hat{\mathbf{D}}=\left(\begin{array}{c}
-\mathbf{D}_{1} \\
\mathbf{D}_{2}
\end{array}\right)
$$

Then

$$
\hat{\mathbf{D}}=\left(\begin{array}{cc}
-\mathbf{I} & \mathbf{0} \\
\mathbf{0} & \mathbf{I}
\end{array}\right) \mathbf{D}
$$

and we find that the corresponding $\hat{\mathbf{E}}$ is

$$
\begin{aligned}
\hat{\mathbf{E}}=\mathbf{P}(\omega) \hat{\mathbf{D}} & =\mathbf{P}(\omega)\left(\begin{array}{cc}
-\mathbf{I} & \mathbf{0} \\
\mathbf{0} & \mathbf{I}
\end{array}\right) \mathbf{D}=\mathbf{P}(\omega)\left(\begin{array}{cc}
-\mathbf{I} & \mathbf{0} \\
\mathbf{0} & \mathbf{I}
\end{array}\right) \mathbf{P}(\omega)^{-1} \mathbf{E} \\
& =\left(\begin{array}{cc}
\mathbf{0} & -\omega^{-2} \mathbf{I} \\
-\omega^{2} \mathbf{I} & \mathbf{0}
\end{array}\right) \mathbf{E} .
\end{aligned}
$$

The same relationship holds between $\hat{\boldsymbol{\varepsilon}}$ and $\boldsymbol{\varepsilon}$. Thus

$$
\hat{\mathbf{E}}_{1}=-\omega^{-2} \mathbf{E}_{2}, \quad \hat{\mathbf{E}}_{2}=-\omega^{2} \mathbf{E}_{1}, \quad \hat{\boldsymbol{\varepsilon}}_{1}=-\omega^{-2} \boldsymbol{\varepsilon}_{2}, \quad \hat{\boldsymbol{\varepsilon}}_{2}=-\omega^{2} \boldsymbol{\varepsilon}_{1} .
$$

Since the matrix $\mathbf{B}_{0}$ of the original boundary form (3.02) can be represented as (cf. (3.08))

$$
\mathbf{B}_{0}=\left(\begin{array}{ll}
\mathbf{0} & \mathbf{J} \\
\mathbf{J} & \mathbf{0}
\end{array}\right)
$$

and since $\mathbf{J}^{*}=-\mathbf{J}$, the symmetry condition in the $\hat{\mathbf{w}}$ variables is

$$
\mathbf{0}=\mathbf{E}_{1}^{*} \mathbf{J E} \mathbf{E}_{2}+\mathbf{E}_{2}^{*} \mathbf{J} \mathbf{E}_{1}=\mathbf{E}_{1}^{*} \mathbf{J} \mathbf{E}_{2}-\mathbf{E}_{2}^{*} \mathbf{J}^{*} \mathbf{E}_{1} \text {, }
$$

from which we conclude not only the fact that $\mathbf{E}_{1}^{*} \mathbf{J E}_{2}$ should be symmetric but also that a spanning matrix for the boundary functional coefficients corresponding to the boundary space spanning matrix is

$$
\mathbf{E}^{\#}=\left[\mathbf{E}_{2}^{*} \mathbf{J}, \mathbf{E}_{1}^{*} \mathbf{J}\right]
$$

(i.e., if $\mathrm{e}^{\#}$ is a linear combination of the rows of this matrix then one of the applicable boundary conditions at $x=0$ is $\mathbf{e}^{\#} \mathbf{w}=0$ ). Now, we verify immediately that if we make the substitutions (3.17) in (3.18) the equation is invariant. Similar conditions apply to the matrix $\varepsilon$ at $x=\pi$.

What this means is the following. Let us denote the fourth derivative operator with boundary spaces $\hat{\mathbf{E}}$ and $\hat{\boldsymbol{\varepsilon}}$ by $\hat{A}$. The corresponding $\hat{\mathbf{E}}^{\#}$ (cf. (3.19)) may then be taken to be (note that the $\mathbf{E}^{\#}$ matrices are only determinate modulo left multiplication by a nonsingular $2 \times 2$ matrix)

$$
\hat{\mathbf{E}}^{\#}=\left[\omega^{4} \mathbf{E}_{1}^{*} \mathbf{J}, \mathbf{E}_{2}^{*} \mathbf{J}\right] \text {. }
$$

That is, compared with $\mathbf{E}^{\#}$, the first and second columns are replaced by the third and fourth, and vice versa. Then the new first and second columns are multiplied by $\omega^{4}$. The fact that the boundary form remains zero means that $\hat{A}$, corresponding to boundary conditions whose coefficients are supplied by linear combinations of the rows of $\hat{\mathbf{E}}^{\#}$, remains a symmetric operator on the new domain.

It is worthwhile to note what happens to the energy form. In view of (1.07) it takes, at $x=0$, the form

$$
\hat{\mathbf{w}}^{*} \mathbf{C}_{0} \hat{\mathbf{w}}=\mathbf{z}^{*} \mathbf{E}^{*} \mathbf{C}_{0} \mathbf{E z}
$$


for the original operator $A$, and since

$$
\mathbf{E}^{*} \mathbf{C}_{0} \mathbf{E}=\mathbf{E}_{1}^{*} \mathbf{J E}_{2}-\mathbf{E}_{2}^{*} \mathbf{J E}_{1}=2 \mathbf{E}_{1}^{*} \mathbf{J E}_{2},
$$

we see that natural boundary conditions, which are to apply for $A$, correspond to the nonnegativity of $\mathbf{E}_{1}^{*} \mathbf{J E} \mathbf{E}_{2}$, already seen to be symmetric. Making the replacements indicated by (3.17), however, we see that the corresponding form for $\hat{A}$ is

$$
\omega^{4} \mathbf{E}_{2}^{*} \mathbf{J} \mathbf{E}_{1}=-\omega^{4} \mathbf{E}_{2}^{*} \mathbf{J}^{*} \mathbf{E}_{1}=-\omega^{4} \mathbf{E}_{1}^{*} \mathbf{J} \mathbf{E}_{2},
$$

and hence must be nonpositive. The corresponding form, involving the $\varepsilon$ matrices, and applying at $x=\pi$, is nonpositive for the original operator $A$ and nonnegative for $\hat{A}$. Thus $\hat{A}$ is supplied with dual natural boundary conditions as defined in $\S 1$.

We have observed in $\S 1$ that $\mathbb{P} \varphi_{k}=\psi_{k}$, where (cf. (1.14))

$$
\psi_{k}(x)=-\gamma^{+} e^{\omega(x-\pi)}-\gamma^{-} e^{-\omega x}+\gamma^{c} \cos \omega x+\gamma^{s} \sin \omega x=\omega_{k}^{-1} D \varphi_{k} .
$$

Since the fourth derivative of $\psi_{k}$ is $\lambda_{k} \psi_{k}=\omega_{k}^{4} \psi_{k}$, our work above shows that these functions are, for $k=1,2,3, \ldots$, eigenfunctions of $\hat{A}$ corresponding to the eigenvalue $\lambda_{k}$, just as the $\varphi_{k}$ are for the original operator $A$. Since we have seen the operator $\hat{A}$ to be symmetric, the $\psi_{k}$ are, consequently, mutually orthogonal, just as the $\varphi_{k}$ are. Since we have seen in $\S 1$ that

$$
\begin{aligned}
\int_{0}^{\pi} \psi_{k}(x)^{2} d x & =\frac{1}{\omega_{k}^{4}} \int_{0}^{\pi} \varphi_{k}^{\prime \prime}(x)^{2} d x \\
& =\frac{1}{\omega_{k}^{4}}\left\{\left(\varphi_{k}, A \varphi_{k}\right)-\left.\varphi_{k}^{*} C_{0} \varphi_{k}\right|_{0} ^{\pi}\right\} \\
& \leq\left(\varphi_{k}, \varphi_{k}\right)=1,
\end{aligned}
$$

we see that $\left\|\psi_{k}\right\| \leq 1$ for all $k$. This, combined with their mutual orthogonality, shows that $\mathbb{P}$ is bounded and that, indeed, $\|\mathbb{P}\| \leq 1$. In the SDE case $\mathbb{P}$ is an isometry (an easy example of the latter occurs in the case where $A$ is the fourth-order operator with boundary conditions $w=w^{\prime}=0$ at both ends, corresponding to a clamped beam in the physical situation; here $\hat{A}$ turns out to be the operator with boundary conditions $w^{\prime \prime}=w^{\prime \prime \prime}=0$ at both ends, corresponding to a free beam; more examples are given in $\S 4$ ).

Invertibility of the operator $\mathbb{P}$ on its range. Since the $\psi_{k}$ span the range of $\mathbb{P}$ and are mutually orthogonal, to show the invertibility of $\mathbb{P}$ on its range it is enough to show that there is a positive number $M$ such that

$$
\left\|\psi_{k}\right\| \geq \mathbf{M}\left\|\varphi_{k}\right\|=\mathbf{M}, \quad k=1,2,3, \ldots
$$

Then we will have $\mathbb{P}^{-1}=\mathbb{Q}$ on the range of $\mathbb{P}$ with $\|\mathbb{Q}\| \leq \mathbb{M}^{-1}$. Since the norms of $e^{-\omega x}$ and $e^{\omega(x-\pi)}$ in $L^{2}[0, \pi]$ are $\sigma^{\prime}\left(\omega^{1 / 2}\right)$ as $\omega \rightarrow \infty$, it is casy to sec that an inequality of the form (3.23) must obtain if we can demonstrate the existence of a positive number $K$ such that

$$
\left(\gamma^{+}\right)^{2}+\left(\gamma^{-}\right)^{2} \leq \mathbb{K}\left[\left(\gamma^{c}\right)^{2}+\left(\gamma^{\prime}\right)^{2}\right]
$$

in all cases under consideration. Since the coefficients $\gamma$ depend on the (assumed natural) boundary conditions stipulated for the operator $A$, it is enough to give a 
complete classification of all natural boundary conditions, perhaps a useful exercise just for its own sake, and show that a bound of the form (3.24) is, indeed, valid in all cases.

The inequality (3.24) is also used for the alternate proof of the boundedness of $\mathbb{P}$ given in $\S 2$.

Classification of the natural boundary conditions for A. Using the same notation as in (3.18), we require that

$$
\mathbf{E}_{1}^{*} \mathbf{J E}_{2}=\mathbf{R}
$$

where, treating only the situation at $x=0$ for the present, $\mathbf{R}$ is a symmetric and nonnegative $2 \times 2$ matrix. Since the matrix $\mathbf{E}$ is only determined modulo right multiplication by a nonsingular $2 \times 2$ matrix, we may replace $\mathbf{R}$ by $\mathbf{S}^{*} \mathbf{R S}=\mathbb{R}$, where

Case (a): $\mathbb{R}=\mathbf{I}$, the $2 \times 2$ identity;

Case (b): $\mathbb{R}=\left(\begin{array}{ll}1 & 0 \\ 0 & 0\end{array}\right)$;

Case $(\mathrm{c}): \mathbb{R}=\left(\begin{array}{ll}0 & 0 \\ 0 & 1\end{array}\right)$;

Case $(\mathrm{d}): \mathbb{R}=\mathbf{0}$.

We study these cases individually.

In Case (a), we first of all represent the lower half of $\mathbf{E}$ in the form

$$
\mathbf{E}_{2}=\left(\begin{array}{ll}
a & b \\
c & d
\end{array}\right)
$$

and an arbitrary $2 \times 2$ rotation matrix as

$$
\mathbf{U}=\left(\begin{array}{cc}
\cos \theta & -\sin \theta \\
\sin \theta & \cos \theta
\end{array}\right)
$$

computing that

$$
\mathbf{E}_{2} \mathbf{U}=\left(\begin{array}{ll}
a \cos \theta+b \sin \theta & b \cos \theta-a \sin \theta \\
c \cos \theta+d \sin \theta & d \cos \theta-c \sin \theta
\end{array}\right) .
$$

Choosing $\theta$ so that $b \cos \theta-a \sin \theta=0$, we may assume that $\mathbf{E}_{2} \mathbf{U}$ is lower triangular. Clearly we still have

$$
\mathbf{U}^{*} \mathbf{E}_{1}^{*} \mathbf{J} \mathbf{E}_{2} \mathbf{U}=\mathbf{U}^{*} \mathbf{U}=\mathbf{I}
$$

Let

$$
\mathbf{E}_{2}=\left(\begin{array}{cc}
r & 0 \\
q & s
\end{array}\right) ; \quad \mathbf{E}_{2}^{-1}=\frac{1}{r s}\left(\begin{array}{cc}
s & 0 \\
-q & r
\end{array}\right) .
$$

Multiplying (3.25) on the right by $\mathbf{E}_{2}^{-1} \mathbf{J}$ and noting that $\mathbf{J}^{2}=-\mathbf{I}$, we see that

$$
\mathbf{E}_{1}^{*}=-\mathbf{E}_{2}^{-1} \mathbf{J} ; \quad \mathbf{E}_{1}=\left(\begin{array}{cc}
0 & -s^{-1} \\
r^{-1} & -q(r s)^{-1}
\end{array}\right) .
$$

Thus, in this case,

$$
\mathbf{E}=\left(\begin{array}{cc}
0 & -s^{-1} \\
r^{-1} & -q(r s)^{-1} \\
r & 0 \\
q & s
\end{array}\right)
$$

where $r$ and $s$ are arbitrary nonzero numbers. For the corresponding case at $x=\pi$, we replace $\mathbf{I}$ above by $-\mathbf{I}$. This has the effect of changing the sign of the upper half of $\mathbf{E}$. 
In Case (b) it is clear that either $\mathbf{E}_{1}$ or $\mathbf{E}_{2}$, perhaps both, are singular. Assuming it is $\mathbf{E}_{2}$, we may suppose that

$$
\mathbf{E}_{2}=\left(\begin{array}{ll}
r \cos \theta & s \cos \theta \\
r \sin \theta & s \sin \theta
\end{array}\right)
$$

and then

$$
\mathbf{J E}_{2}=\left(\begin{array}{cc}
-r \sin \theta & -s \sin \theta \\
r \cos \theta & s \cos \theta
\end{array}\right) .
$$

From the form of $\mathbf{E}_{1}^{*} \mathbf{J E}_{2}$, the first row of $\mathbf{E}_{1}^{*}$ must have the form $(q \cos \theta \quad q \sin \theta)$. Since the columns of $\mathbf{J E}_{2}$ are dependent, the only condition permitting us to define the second row of $\mathbf{E}_{1}^{*}$ in such a way that the $(2,1)$ entry of $\mathbf{E}_{1}^{*} \mathbf{J} \mathbf{E}_{2}$ is zero while the $(2,2)$ entry is one, is that $r$ should be zero while $s$ is not. So

$$
\mathbf{E}_{2}=\left(\begin{array}{cc}
0 & s \cos \theta \\
0 & s \sin \theta
\end{array}\right), \quad s \neq 0 .
$$

On the other hand,

$$
\mathbf{E}_{1}^{*}=\left(\begin{array}{cc}
q \cos \theta & q \sin \theta \\
\alpha \cos \theta-s^{-1} \sin \theta & \alpha \sin \theta+s^{-1} \cos \theta
\end{array}\right)
$$

so that

$$
\mathbf{E}_{1}=\left(\begin{array}{ll}
q \cos \theta & \alpha \cos \theta-s^{-1} \sin \theta \\
q \sin \theta & \alpha \sin \theta+s^{-1} \cos \theta
\end{array}\right) .
$$

Since the rank of $\mathbf{E}$ must be two, $q \neq 0$. Multiplying $\mathbf{E}$ on the right by an appropriate nonsingular $2 \times 2$ matrix we can subtract a multiple of the first column of $\mathbf{E}$ from the second and finally arrive at

$$
\mathbf{E}=\left(\begin{array}{cc}
q \cos \theta & -s^{-1} \sin \theta \\
q \sin \theta & s^{-1} \cos \theta \\
0 & s \cos \theta \\
0 & s \sin \theta
\end{array}\right), \quad q \neq 0, \quad s \neq 0 .
$$

If, on the other hand, we suppose that it is $\mathbf{E}_{1}$ which is singular, transposing and noting that $\mathbf{J}^{*}=-\mathbf{J}$, we have

$$
-\mathbf{E}_{2}^{*} \mathbf{J E} \mathbf{E}_{1}=\mathbf{I}
$$

so that we may take $\mathbf{E}_{2}$ in the role of the previous $\mathbf{E}_{1},-\mathbf{E}_{1}$ in the role of the previous $\mathbf{E}_{2}$, this gives

$$
E=\left(\begin{array}{cc}
0 & s \cos \theta \\
0 & s \sin \theta \\
q \cos \theta & s^{-1} \sin \theta \\
q \sin \theta & -s^{-1} \cos \theta
\end{array}\right)
$$

In Case (c) we multiply the indicated equation on the right and on the left by

$$
\mathbf{L}=\left(\begin{array}{ll}
0 & 1 \\
1 & 0
\end{array}\right)
$$

to get

$$
\mathbf{L}^{*} \mathbf{E}_{1}^{*} \mathbf{J} \mathbf{E}_{2} \mathbf{L}=\left(\begin{array}{cc}
0 & 0 \\
0 & 1
\end{array}\right)
$$


and we see that this is not really a separate case from (b).

Case (d) naturally divides into three subcases. The first two arise for $\mathbf{E}_{1}=\mathbf{0}, \mathbf{E}_{2}$ nonsingular and $\mathbf{E}_{2}=\mathbf{0}, \mathbf{E}_{1}$ nonsingular, the nonsingularity in each case following from the requirement that $\mathbf{E}$ should have rank two. The only remaining possibility is readily seen to be that both $\mathbf{E}_{1}$ and $\mathbf{E}_{2}$ each have rank one. The arguments leading to (3.26), (3.27) may be repeated and we subsequently conclude that $\mathbf{E}_{l}$ has the same form, i.e.,

$$
\mathbf{E}_{1}=\left(\begin{array}{cc}
\rho \cos \theta & \sigma \cos \theta \\
\rho \sin \theta & \sigma \sin \theta
\end{array}\right)
$$

Thus

$$
\mathbf{E}=\left(\begin{array}{cc}
\rho \cos \theta & \sigma \cos \theta \\
\rho \sin \theta & \sigma \sin \theta \\
r \cos \theta & s \cos \theta \\
r \sin \theta & s \sin \theta
\end{array}\right)
$$

Assuming, without loss of generality, that $\rho \neq 0$, we may multiply $\mathbf{E}$ on the right by a nonsingular matrix which has the effect of subtracting a multiple of the first column from the second in such a way that, renaming the coefficients, we may assume $\sigma=0$. The rank condition on $\mathbf{E}$ then implies that $s \neq 0$ and, subtracting a multiple of the second column from the first, we may replace $r$ by zero so that finally (renaming $\rho$ as $r$ for alphabetic consistency)

$$
\mathbf{E}=\left(\begin{array}{cc}
r \cos \theta & 0 \\
r \sin \theta & 0 \\
0 & s \cos \theta \\
0 & s \sin \theta
\end{array}\right)
$$

Proof of (3.24). The spanning matrices for the boundary conditions, expressed in "linear functional" form, are found by using the symmetry condition for the form (3.02). Expressing $\hat{\mathbf{w}}$ and $\hat{\mathbf{v}}$ in a manner consistent with (3.16), the symmetry condition is $(3.18)$, which is equivalent to

$$
\left[\mathbf{E}_{2}^{*} \mathbf{J}, \mathbf{E}_{1}^{*} \mathbf{J}\right]\left(\begin{array}{l}
\mathbf{E}_{1} \\
\mathbf{E}_{2}
\end{array}\right)=\mathbf{0}
$$

so that the boundary conditions themselves take the form

$$
\left[\mathbf{E}_{2}^{*} \mathbf{J}, \mathbf{E}_{\mid}^{*} \mathbf{J}\right] \hat{\mathbf{w}}=\mathbf{0},
$$

with a similar condition applying to $\hat{\mathbf{v}}$, of course. The transformed boundary conditions in the $\tilde{\mathbf{w}}, \tilde{\mathbf{v}}$ variables then correspond, in linear functional form, to the equations

$$
\boldsymbol{\varphi}(\omega) \tilde{\mathbf{w}}=\left[\mathbf{E}_{2}^{*} \mathbf{J}, \mathbf{E}_{1}^{*} \mathbf{J}\right] \mathbf{P}(\omega) \tilde{\mathbf{w}}=\mathbf{0}
$$

and a similar condition on $\tilde{\mathbf{v}}$. In the ${ }^{\sim}$ coordinate system the eigenfunctions $\dot{\varphi}$ have the component values

$$
\tilde{\boldsymbol{\varphi}}(0)=\left(\begin{array}{c}
\gamma^{-} \\
\gamma^{+} \\
\left(\gamma^{c}-\gamma^{s}\right) / 2 \\
\left(\gamma^{c}+\gamma^{s}\right) / 2
\end{array}\right)
$$


From this and the form (3.30), together with the classification of natural boundary conditions obtained above, we will show that $\gamma^{-}$is bounded in terms of $\gamma^{c}$ and $\gamma^{s}$ in every case. A completely symmetric situation exists at $x=\pi$ in respect to $\gamma^{+}$, so that will not require additional treatment.

We take the eigenfunction $\varphi(x)$ in the form (3.13). Given a set of natural boundary conditions at $x=0$ we establish that

$$
\left|\gamma^{-}\right| \leq M_{0}\left|\gamma^{+}\right| e^{-\omega \pi}+N_{0}\left(\left|\gamma^{c}\right|+\left|\gamma^{s}\right|\right)
$$

with $M_{0}$ and $N_{0}$ positive and independent of $\omega$. Another natural boundary condition applies at $x=\pi$ and yields

$$
\left|\gamma^{+}\right| \leq M_{\pi}\left|\gamma^{-}\right| e^{-\omega \pi}+N_{\pi}\left(\left|\gamma^{c}\right|+\left|\gamma^{s}\right|\right) .
$$

Then since the matrix

$$
\left(\begin{array}{cc}
1 & -M_{0} e^{-(\omega \pi} \\
-M_{\pi} e^{-\omega \pi} & 1
\end{array}\right)
$$

tends to the $2 \times 2$ identity as $\omega \rightarrow \infty$, we have the desired result. Since the situations at $x=0$ and at $x=\pi$ are, as we have remarked, symmetric, it will be enough to prove that (3.31) holds for all possible natural boundary conditions imposed at $x=0$. If we let the entries of the matrix in (3.30) be denoted by $\varphi_{j}^{i}(\omega), i=1,2, j=1,2,3,4$, then what needs to be shown is that there is a value of $i$ such that

$$
\left|\varphi_{1}^{i}(\omega) / \varphi_{j}^{i}(\omega)\right| \leq \mu, \quad j=2,3,4,
$$

with $\mu$ independent of $\omega$ for large values of $\omega$.

We begin with Case (a). From the form of $\mathbf{P}(\omega)$ (cf. (3.04)) and the fact that $\mathbf{E}$ and $\mathbf{J}$ are independent of $\omega$, it is clear that no entry of $\varphi(\omega)$ grows faster than $\omega^{3}$. A simple computation shows that the first column of $\varphi(\omega)$ is

$$
\varphi_{1}(\omega)=\frac{1}{2}\left(\begin{array}{c}
q+r \omega+r^{-1} \omega^{2} \\
s-(r s)^{-1} q \omega^{2}-s^{-1} \omega^{3}
\end{array}\right)
$$

Since $s \neq 0$, the presence of the term $-s^{-1} \omega^{3}$ in (3.34) guarantees that (3.33) holds for $i=2$, completing the analysis.

In Case (b) with $\mathbf{E}$ in the form (3.28) we compute the first row of $\varphi(\omega)$ :

$$
\boldsymbol{\varphi}^{1} \omega=\left((q \sin \theta) \omega^{2}+(q \cos \theta) \omega^{3}, \quad q \sin \theta-(q \cos \theta) \omega^{3} .\right.
$$

$$
\left.(q \sin \theta) \omega^{2} . \quad(-q \cos \theta) \omega^{3}\right) .
$$

Since $q \neq 0$, when $\cos \theta \neq 0$ the first entry includes a term which grows like $\omega^{3}$ and there is no problem. If $\cos \theta=0$, then no entries involve cubic terms and since $|\sin \theta|=1$, the first entry includes a term growing like $\omega^{2}$, from which (3.32) is then easily verified. On the other hand, when $\mathbf{E}$ is in the form (3.29) then

$\boldsymbol{\varphi}^{1}(\omega)=(q \sin \theta+(q \cos \theta) \omega, \quad q \sin \theta-(q \cos \theta) \omega, \quad q \sin \theta, \quad q \sin \theta+(q \cos \theta) \omega)$ and the analysis is entirely similar.

As we have remarked earlier, Case (c) is really the same as (b).

The argument in Case (d) is entirely the same as in Cases (b), (c), but even simpler; one may use either the first or the second row of $\varphi(\omega)$ to obtain the result. 
We remark that the case wherein $\mathbf{E}_{1}=0$, which occurs only under (d) above, is what we have referred to in $\S 1$ as the trigonometric case, since the real exponential parts of the eigenfunctions reduce to zero when this occurs.

Finally, a word about the equation from which the eigenvalues, and hence the spatial frequency coefficients $\omega$, may be determined. We have noted that, using the boundary conditions which apply at $x=0$, we can always solve for $\gamma^{-}$. If the expression for $\gamma^{-}$so obtained is substituted into the other, linearly independent, boundary condition which applies at $x=0$, an equation is obtained involving $\gamma^{+}, \gamma^{c}, \gamma^{s}$, the trigonometric expressions $\sin \omega \pi, \cos \omega \pi$, and $e^{-(1) \pi}$. Using the boundary conditions applying at $x=\pi$ the coefficient $\gamma^{+}$can be eliminated similarly and one obtains, in the end, two equations involving $\gamma^{c}$ and $\gamma^{s}$ together with $\cos \omega \pi$, $\sin \omega \pi$, and $e^{-(j) \pi}$. The determinant of this last pair of equations in $\gamma^{c}$ and $\gamma^{s}$ provides a trigonometric/exponential equation wherein the exponential terms are asymptotically small as $\omega$ tends to infinity. From this equation the numbers $\omega_{k}$ (hence the eigenvalues $\lambda_{k}$ ) can be determined and may be shown to have the form indicated in (1.18).

4. Examples, remarks, etc. Probably the most significant contribution which we can make, example-wise, is to clarify the relationship between the original fourthorder operator $A$, assumed to be supplied with natural boundary conditions, and the corresponding operator $\hat{A}$ with what we have called "dual natural" boundary conditions.

Let us consider a cantilever beam with elastic forces applying at the free end, $x=\pi$. Assuming $\rho=E I=1$, the boundary conditions become

$$
\begin{aligned}
w(0, t) & =0, & & \frac{\partial w}{\partial x}(0, t)=0, \\
\frac{\partial^{3} w}{\partial x^{3}}(\pi, t)-\alpha w(\pi, t) & =0, & & \frac{\partial^{2} w}{\partial x^{2}}(\pi, t)+\beta \frac{\partial w}{\partial x}(\pi, t)=0,
\end{aligned}
$$

where $\alpha$ and $\beta$ are nonnegative real numbers. Then the boundary space spanning matrix (3.19) becomes, at $x=0$,

$$
\mathbf{E}^{\#}=\left(\begin{array}{llll}
1 & 0 & 0 & 0 \\
0 & 1 & 0 & 0
\end{array}\right)
$$

and the boundary space spanning matrix at $x=\pi$ can be taken to be

$$
\boldsymbol{\varepsilon}^{\#}=\left(\begin{array}{cccc}
-\alpha & 0 & 0 & 1 \\
0 & \beta & 1 & 0
\end{array}\right) \text {. }
$$

Still making use of that formula, we conclude that

$$
\mathbf{E}=\left(\begin{array}{cc}
0 & 0 \\
0 & 0 \\
0 & -1 \\
1 & 0
\end{array}\right), \quad \boldsymbol{\varepsilon}=\left(\begin{array}{cc}
-1 & 0 \\
0 & 1 \\
0 & -\beta \\
-\alpha & 0
\end{array}\right)
$$

From 3.20 we see that the corresponding spanning matrices for the boundary conditions of the dual problem are

$$
\hat{\mathbf{E}}^{\#}=\left[\omega^{4} \mathbf{E}_{1}^{*} \mathbf{J}, \mathbf{E}_{2}^{*} \mathbf{J}\right]=\left(\begin{array}{cccc}
0 & 0 & 1 & 0 \\
0 & 0 & 0 & 1
\end{array}\right)
$$


and

$$
\hat{\boldsymbol{\varepsilon}}^{\#}=\left[\omega^{4} \boldsymbol{\varepsilon}_{1}^{*} \mathbf{J}, \boldsymbol{\varepsilon}_{2}^{*} \mathbf{J}\right]=\left(\begin{array}{cccc}
0 & \omega^{4} & -\alpha & 0 \\
\omega^{4} & 0 & 0 & \beta
\end{array}\right) .
$$

From this we see that the dual natural boundary conditions in this case are, at $x=0$,

$$
\frac{\partial^{2} w}{\partial x^{2}}(0, t)=0, \quad \frac{\partial^{3} w}{\partial x^{3}}(0, t)=0,
$$

which are those corresponding to a free endpoint, and at $x=\pi$,

$$
\begin{gathered}
\omega^{4} \frac{\partial w}{\partial x}(\pi, t)-\alpha \frac{\partial^{2} w}{\partial x^{2}}(\pi, t)=0 \\
\omega^{4} w(\pi, t)+\beta \frac{\partial^{3} w}{\partial x^{3}}(\pi, t)=0
\end{gathered}
$$

When $\alpha$ and $\beta$ are zero, in which case the original system has a free end at $x=\pi$, the dual system has a clamped end there. If both $\alpha$ and $\beta$ are nonzero, so that the original system has elastic boundary conditions of the standard sort at $x=\pi$, we see that the dual system also has elastic boundary conditions at $x=\pi$ but with the $\alpha$ and $\beta$ of (4.02) replaced by $-\alpha^{-1} \omega^{4}$ and $-\beta^{-1} \omega^{4}$, respectively. Thus in the dual problem the boundary elasticities act so as to render the equilibrium $w=0$ unstable and we may expect negative eigenvalues $\lambda$ to appear. The frequency dependence signalled by the appearance of the factor $\omega^{4}$ also introduces a new feature. Since $\omega^{4} \psi(x, t)=\lambda \psi(x, t)=\frac{\partial^{2} \psi}{\partial t^{2}}(x, t)$ for the corresponding eigenfunction solutions $\psi(x, t)=e^{ \pm i \omega^{2} t} \psi(x)$ of the beam equation with these boundary conditions, the boundary conditions $(4.04)$ can be reinterpreted as

$$
\begin{aligned}
& \frac{\partial^{3} w}{\partial t^{2} \partial x}(\pi, t)-\alpha \frac{\partial^{2} w}{\partial x^{2}}(\pi, t)=0 \\
& \frac{\partial^{2} w}{\partial t^{2}}(\pi, t)+\beta \frac{\partial^{3} w}{\partial x^{3}}(\pi, t)=0
\end{aligned}
$$

so that the "elastic feedback" acts not on the slope and displacement at $x=\pi$ but rather on the time rate of acceleration of these quantities. These can also be interpreted in terms of tip masses and tip moments of inertia.

Now let us consider the range of the operator $\mathbb{P}$, spanned by those eigenfunctions $\psi$ of $\hat{A}$ for which $\lambda$ is positive. As we have seen, these are the images under $\mathbb{P}$ of the eigenfunctions $\varphi$ of $A$ corresponding to positive eigenvalues $\lambda$. The negative eigenvalues and eigenfunctions of $\hat{A}$ cannot be related to similar eigenvalues and eigenfunctions of $A$ because those eigenfunctions $\psi$ of $\hat{A}$ do not have the form indicated in (1.14) and thus are not images of corresponding $\varphi$, of the form (1.10), under $\mathbb{P}$. The range of $\mathbb{P}$ thus does not include $\psi$ corresponding to negative $\lambda$, and, in such cases therefore, is not the whole space $L^{2}[0, \pi]$.

Let us agree to denote by $\hat{A}^{1 / 2}$ the positive square root of $\hat{A}$, restricted to the range, $\mathscr{R}$, of $\mathbb{P}$, the closed subspace of $L^{2}[0, \pi]$ spanned by the eigenfunctions $\psi$ of $\hat{A}$ which correspond to positive eigenvalues $\lambda$ of that operator. Then we have the following 
diagram to explain the relationships between $A^{1 / 2},-D^{2}$, and $\hat{A}^{1 / 2}$.

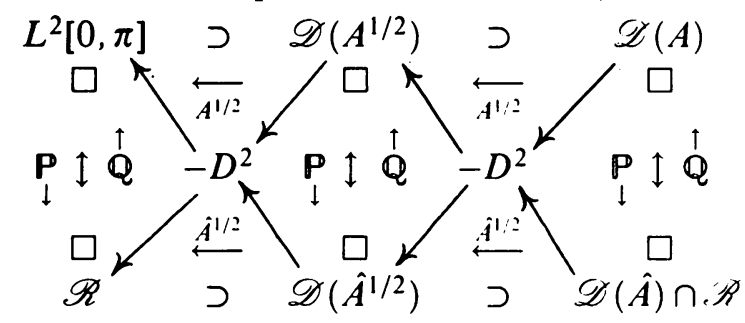

Since the eigenfunctions $\varphi_{k}$ of the operator $A$ form an orthonormal basis for $L^{2}[0, \pi]$, it is easy to see that the operator $P$ defined in (1.16) has the formal representation

$$
\begin{aligned}
(P w)(x) & =\sum_{k=1}^{\infty}\left(w, \varphi_{k}\right)_{L^{2}[0, \pi]} \theta_{k}(x) \\
& =\int_{0}^{\pi}\left(\sum_{k=1}^{\infty} \theta_{k}(x) \varphi_{k}(\xi)\right) u(\xi) d \xi \\
& \equiv \int_{0}^{\pi} \mathrm{P}(x, \xi) u(\xi) d \xi
\end{aligned}
$$

where for $\lambda_{k} \neq 0$,

$$
\theta_{k}(x)=-2\left(\gamma_{k}^{+} e^{\left(\omega_{k}(x-\pi)\right.}+y_{k}^{-} e^{-(1)_{k} \cdot x}\right)
$$

and $\theta_{1}=-\varphi_{1}$ if $\lambda_{1}=0$. The formally defined kernel $\mathrm{P}(x, \xi)$ is readily seen to be convergent for $0<x<\pi$; there will, in general, be singularities at the boundaries $x=0$ and $x=\pi$. Thus we have the representation, for $u \in \mathscr{Z}\left(A^{1 / 2}\right)$,

$$
\left(-D^{2} w\right)(x)=\left(A^{1 / 2} w\right)(x)+\int_{0}^{\pi} \mathrm{P}(x, \xi)\left(A^{1 / 2} w\right)(\xi) d \xi .
$$

A similar representation, also valid for $w \in \mathscr{D}\left(A^{1 / 2}\right)$ (which we have also taken to be the domain of $-D^{2}$ in this paper),

$$
\left(A^{1 / 2} w\right)(x)=-\left(D^{2} w\right)(x)-\int_{0}^{\pi} \mathrm{Q}(x, \xi)\left(D^{2} w\right)(\xi) d \xi
$$

can be obtained by representing $\mathbb{Q}$ (cf. (1.20)) in the form $I+Q$ and noting that on the range of $\mathbf{P}=$ range of $D^{2}$,

$$
(Q w)(x)=-\sum_{\lambda_{k} \neq 0} \frac{\left(w, \psi_{k}\right)}{\left\|\psi_{k}\right\|} \theta_{k}(x)
$$

Formula (4.07) exhibits $A^{1 / 2} w$ more or less explicitly as a transform of $-D^{2} w$. It seems likely that careful study of the kernels $\mathrm{P}(x, \xi)$ and $\mathrm{Q}(x, \xi)$ in (4.06) and (4.07) might yield additional useful information about the relationship between the operators $A^{1 / 2}$ and $-D^{2}$.

\section{REFERENCES}

[1] F. V. Atkinson, Discrete and Continuous Boundary I'alue Prohle'ms. Vol. 8 of Mathematics in Science and Engineering, Academic Press, New York, 1964

[2] G. Chen and D. L. Russell, A mathematical model for linear clastic systems with structural clamping. Quart. Appl. Math. 39, 433-454 (1982) 
[3] P. L. Duren, Theory of $H^{p}$ Spaces, Academic Press, New York, 1970

[4] G. H. Hardy, J. E. Littlewood, and G. Pòlya, Inequalities, Cambridge University Press. 1934

[5] L. F. Ho and D. L. Russell, Admissible input elements for systems in Hilhert space and a Carleson measure criterion, SIAM J. Control Optim. 21, 614-640 (1983)

[6] T. Kato, Perturbation Theory for Linear Operators, Springer-Verlag, New York. 1966

[7] S. Kaczmarz and H. Steinhaus, Theorie der Orthogonalreihen, Monografje Matematyczne. Tom VI: Warsaw, 1935

[8] R. S. Phillips, Dissipative hyperbolic systems, Trans. Amer. Math. Soc. 86, 109-173 (1957)

[9] S. C. Power, Hankel Operators on Hilbert Space, Research Notes in Mathematics. Vol. 64. Pitman Advanced Publishing Program, Boston, London, Melbourne, 1982

[10] W. T. Reid, Ordinary Differential Equations, John Wiley and Sons, New York. 1971

Reference added in proof. Dr. G. Leugering has very kindly pointed out earlier work related to our boundary value classification scheme.

[11] S. A. Janczewski, Sur quelques problèmes aux limites pour des équations diffiercentielles linćaires ordinaires du quatrieme ordre, Comptes Rendus 184, 141-143 (1927)

Further, related, work by the same author appears in Comptes Rendus 184, 261263 (1927), Comptes Rendus 186, 287-289 (1928), and in Annals of Mathematics (2) 29, 521-542 (1927/28). 